\title{
BMJ Open Health professionals in a changing climate: protocol for a scoping review
}

\author{
Lianping Yang, ${ }^{\oplus}$ Chaojie Liu, ${ }^{2}$ Jeremy Hess, ${ }^{3}$ Dung Phung, ${ }^{4}$ Cunrui Huang ${ }^{1}$
}

To cite: Yang L, Liu C, Hess J, et al. Health professionals in a changing climate: protocol for a scoping review. BMJ Open 2019;9:e024451. doi:10.1136/ bmjopen-2018-024451

- Prepublication history for this paper is available online. To view these files, please visit the journal online (http://dx.doi org/10.1136/bmjopen-2018024451).

Received 26 May 2018 Revised 11 November 2018 Accepted 21 December 2018

A Check for updates

C Author(s) (or their employer(s)) 2019. Re-use permitted under CC BY-NC. No commercial re-use. See rights and permissions. Published by BMJ.

${ }^{1}$ School of Public Health, Sun Yat-sen University, Guangzhou, China

${ }^{2}$ Department of Public Health, La Trobe University, Melbourne, Australia

${ }^{3}$ Departments of Emergency Medicine, Environmental and Occupational Health Sciences, and Global Health, Schools of Medicine and Public Health, University of Washington, Seattle, USA

${ }^{4}$ Centre for Environment and Population Health, Griffith University, Brisbane, Australia

Correspondence to

Cunrui Huang;

huangcr@mail.sysu.edu.cn

\begin{abstract}
Introduction Climate change will impose significant health impacts. Although we know health professionals should play a critical role in protecting human health from climate change, their preparedness to engage with these issues worldwide is unclear. This study aims to map the range and nature of existing evidence regarding health professionals' knowledge, attitudes, perceptions and practices regarding climate change and health impacts and the challenges they face, and identify knowledge gaps to guide future development of research, policy and practices.
\end{abstract}

Methods and analysis We will perform a scoping review based on the six-stage framework proposed by Arksey and 0'Malley. Our study includes peer-reviewed literature focusing on any aspect of health professionals' work regarding climate change and health since 2002 and indexed in MEDLINE/Pubmed, Web of Science, Scopus or Embase. Identified papers will be described and assessed. Thematic analysis will be applied to evaluate and categorise the study findings.

Implications and dissemination This is the first scoping review of health professionals' activities to anticipate and prepare for health impacts attributable to climate change. It will provide evidence regarding the current situations worldwide and gaps in preparedness. The findings can be used to highlight accomplishments to date, identify gaps and further develop good practices for health professionals' engagement. The results will be published in the peer-reviewed literature and shared at health professional society meetings.

\section{INTRODUCTION}

Climate change (or global warming) is one of the major global health concerns of the 21st century. Climate change's impacts are already being observed today worldwide. If no actions are taken, the risk to human health will be unacceptably high and potentially catastrophic. ${ }^{12}$ Climate change poses a range of health threats, many of which have the potential to interact and overlap. Some causal pathways are relatively short and direct (eg, heat waves, storms, floods and forest fires), while some are longer and their health impacts are more indirectly mediated through socioecological systems (eg, agricultural losses and other nutritional impacts and changing patterns of infectious disease)
Strengths and limitations of this study

- The systematic scoping review will fill an important research gap, as evidence regarding health professionals' engagement and preparedness is beginning to accumulate but has not yet been collated and centrally assessed.

- This review will search multidisciplinary databases covering medicine, health, society and the environment in order to ensure a comprehensive assessment of the literature.

- No restrictions will be applied on study type, design, location or health professional role.

- As we aim to synthesise all the different aspects with regard to climate change and health professionals, identified literature will not be excluded based on quality assessment, though the type of study and the strength of available evidence will be noted in the review.

- As full-text review will not apply to the small number of publications in a language other than English and Chinese, the review report may be biased.

and changes in social structure (eg, human migration and community conflicts). The indirect consequences such as ecosystem collapse may drive the most significant health impacts but are more difficult to estimate using currently available methods. The Fifth Assessment Report from the Intergovernmental Panel on Climate Change affirms that 'there is increasing certainty these [impacts] will continue and, in some cases, accelerate'. Significant adverse health impacts are at this point unavoidable and potentially irreversible, and the potential for prevention activities to blunt impacts has been limited by the slow and inadequate response to the changing climate over the past two decades. ${ }^{2}$

Population vulnerability to these threats and thus risk of significant impacts varies by region, raising an important issue of health inequity, both globally from a north-south perspective and across individual societies. Disadvantaged populations such as the elderly, children and those with pre-existing ill health are particularly vulnerable to heat, cold, allergens and air pollution as a result 
of extreme weather events. ${ }^{4}$ The poor and other socially marginalised groups are disproportionally affected by these extreme weather events. They suffer more profoundly from disruptions to the social system such as economic insecurity, displacement, homelessness and conflict. ${ }^{145}$ Researchers believe that the health impacts of climate change are a consequence of environmental justice issues. ${ }^{6}$

Climate change will likely exacerbate lots of environmental health risks familiar to public health professionals and clinical workers, and will create novel hardships and threats in many areas. Health professionals should play critical roles in addressing the health threats related to climate change. ${ }^{78}$ The voice coming from the health profession is vital in raising public awareness and driving political agenda on climate change. In the USA, the general public were most likely to trust their primary care physicians followed by the Centers for Disease Control and Prevention (CDC) in obtaining information about health consequences of global warming. ${ }^{9}$ The health profession can act as advocates for population health by communicating the opportunities and threats to policy makers and the general public. ${ }^{10}$

In general, health professionals can take many different actions, such as advocating for implementation of mitigation measures in the health sector and generally; being vocal in framing climate change as a public health issue; pushing for rapid attainment of the United Nations Sustainable Development goals, and speaking out to protect the vulnerable groups to reduce poverty and inequity related to climate change; advocating for political leadership at the local, national and international levels to reduce the risks of dangerous climate change; promoting the available solutions (eg, renewable energy streams, improved energy efficiency and technology transfer from high-income to middle-income and low-income countries) in the health sector and beyond. ${ }^{10}$ Increasingly, due to the climate change commitment from prior emissions, health professionals must also advocate strongly for adaptation measures in the health sector and other areas of the economy that affect health, such as water and agriculture. The 2016 'WHO conference on climate change and health' concluded with a loud and clear call to the international health community: 'it is imperative that health professionals worldwide show strong leadership in tackling climate change'. The US CDC's Climate Ready States and Cities Initiative represents a useful example for health professionals to move forward and engage in robust, targeted local preparedness and response. ${ }^{11}$

The extent to which health professionals around the world are prepared to act to reduce the likelihood of dangerous climate change and to respond adequately to health impacts, however, is unclear. Therefore, we will conduct a scoping review to fully understand what is known about health professionals' knowledge, attitudes, perceptions and practices regarding climate change and its health impacts.

\section{Research objectives}

In this scoping review, the objectives are to collate published academic literature/papers on health professionals' knowledge, attitudes, perceptions and practices regarding climate change and health impacts. We aim to map the findings by categorising papers according to their topic and context information, establish an open database of relevant literature and provide a thematic analysis on the content. The preparedness of health professionals on climate change actions will be assessed through the gaps in their knowledge, attitudes, perceptions and practices. The organisational, national and international hurdles and challenges that health professionals face in realising their full potential will be identified.

The specific objectives of this study include the following:

- Providing an overview of existing peer-reviewed literature over time about the knowledge, attitudes, perceptions and practices of health professionals regarding climate change and its health impacts.

- Establishing an open database of categorised literature regarding climate change and health professionals' preparedness.

- Making recommendations on the roles of health professionals in climate change and potential areas for future research.

\section{METHOD}

\section{Patient and public involvement}

This is a review study and there is no patient or public involvement.

\section{Scoping review methodology}

The aim of systematic scoping reviews is to rapidly synthesise 'evidence on crucial concepts associated with broad research topics in addition to identifying the central sources and forms of evidence available'. ${ }^{12}$ While methodological frameworks developed by Arksey and O'Malley and enhanced by Levac et $a l^{213}$ allow for more standardisation, scoping review methods 'remain flexible to enable clarification of concepts and research questions following familiarisation with the literature' ${ }^{14}$

Systematic scoping reviews incorporate different study types and designs. It goes beyond answering questions about intervention effectiveness. Rigorous and systematic methods apply to demonstrate credible evidence for synthesising knowledge. This is beneficial when a shortage of evidence prevents conduct of systematic reviews, and when there exists substantial diversity in approaches to the topic. ${ }^{13}$

Undertaking a systematic scoping review will allow the researchers to explore extensively the academic literature on the health professional's knowledge, attitudes, perceptions and practices regarding climate change and health impacts and the challenges they face.

\section{Protocol design}

The review will apply the methodological approach proposed by Arksey and O'Malley and Levac et al. ${ }^{1213}$ They 
Table 1 Research questions for the scoping review on 'climate change and health professionals'

\section{Research questions}

1. What health professional roles have been studied regarding the health impacts of climate change?
Doctors/physicians, public health professionals, nurses, general practitioners, administrators, educators and other health professionals

2. What is the geographical origin and focus of the publications?*

- Africa

- The Americas

- South-East Asia

- Europe

- The Eastern Mediterranean

- The Western Pacific

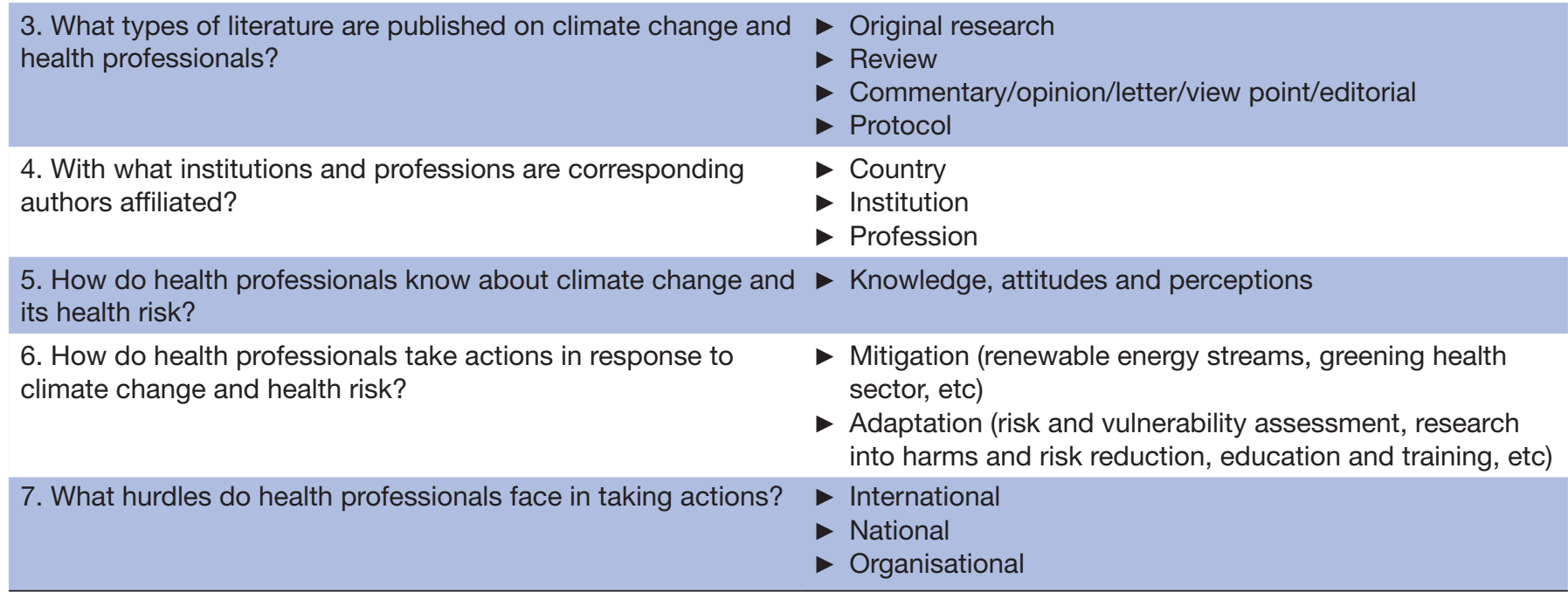

${ }^{*}$ Categorisation of regions by the WHO.

delineated a six-stage framework for operating a scoping review: (1) identifying the research gap and the questions, (2) identifying relevant literature, (3) assessment and selection of studies, (4) charting and presenting the data, (5) collating, summarising and reporting findings and (6) external consultation with relevant stakeholders. ${ }^{12}$

\section{Stage 1: Identifying the research question}

Climate change poses serious threats to population health, and health professionals should play critical roles in combating the health risks. While some members of the health community are highly aware of the issues, we do not know how the health community more generally perceives this problem and what actions it prioritises, illustrating an evidence gap. Therefore, we developed our specific research questions (table 1).

\section{Stage 2: Identifying relevant studies}

Relevant studies will be identified from MEDLINE/ PubMed, Web of Science, Scopus and Embase. The search strategy will include terms broad enough to cover all areas of climate change and health professionals, including Medical Subject Headings (MeSH) terms, subject headings and keywords applied to identify articles. The search strategy in the different databases will be reviewed by a Sun Yat-Sen University medical librarian.

The search strategy will be built based on the keywords demonstrated in table 2. The terms 'preparedness' and

Table 2 Search terms for the scoping review on 'climate change and health professional'

\begin{tabular}{lll} 
Climate change-related terms & Health professionals-related terms & Date of publication \\
\hline Climate change & Health/medical/clinic/public health/healthcare Professional/ & Since 2002 \\
Climate variability & worker/technician/technologist/staff/ & \\
Global warming & practitioner/officer/assistant/student \\
Greenhouse effect/greenhouse gas & Physician/physician assistant \\
emissions (GHGE) & General practitioner/nurse/doctor/intern \\
Extreme weather/heat wave/high & Internist/surgeon/psychiatrist/paediatrician/psychologist/ \\
temperature/drought/flooding & endocrinologist/dentist/odontologist/ \\
& obstetricians/gynaecologists \\
\hline
\end{tabular}


Table 3 Search strategy syntax for different databases

\begin{tabular}{ll}
\hline Database & Search strategy syntax \\
\hline PubMed & (Climate change [Title/Abstract] OR climate variability [Title/Abstract] OR global warming [Title/Abstract] OR \\
& greenhouse effect [Title/Abstract] OR GHGE [Title/Abstract] OR extreme weather [Title/Abstract] OR heat \\
& wave [Title/Abstract] OR high temperature [Title/Abstract] OR drought [Title/Abstract] OR flooding [Title/ \\
& Abstract]) AND ((health [All Fields] OR medical [All Fields] OR clinic [All Fields] OR public health [All Fields] \\
& OR healthcare [All Fields] OR health care [All Fields]) AND (professional [All Fields] OR worker [All Fields] OR \\
& technician [All Fields] OR technologist [All Fields] OR staff [All Fields] OR practitioner [All Fields] OR officer \\
& [All Fields] OR assistant [All Fields] OR student [All Fields]) OR physician [All Fields] OR physician assistant \\
& [All Fields] OR general practitioner [All Fields] OR general doctor [All Fields] OR nurse [All Fields] OR doctor \\
& [All Fields] OR intern [All Fields] OR internist [All Fields] OR surgeon [All Fields] OR paediatrician [All Fields] \\
& OR psychiatrist [All Fields] OR psychologist [All Fields] OR endocrinologist [All Fields] OR dentist [All Fields] \\
& OR odontologist [All Fields]) AND (2002/01/01 [PDAT]: [PDAT])
\end{tabular}

Web of Science $\mathrm{Tl}=$ (climate change OR climate variability OR global warming OR greenhouse effect OR GHGE OR extreme weather OR heat wave OR high temperature OR drought OR flooding) AND TS=((health OR medical OR clinic OR public health OR healthcare OR health care) AND (professional OR worker OR technician OR technologist OR staff OR practitioner OR officer OR assistant OR student) OR physician OR physician assistant OR general practitioner OR general doctor OR nurse OR doctor OR intern OR internist OR surgeon OR psychiatrist OR Pediatrician OR psychologist OR endocrinologist OR dentist OR odontologist) AND TIME SPAN $=(2002-)$

Scopus
OR 'extreme weather' OR 'heat wave' OR drought OR flooding) AND (TITLE-ABS-KEY (health OR medical
OR 'public health' OR healthcare OR clinic) AND TITLE-ABS-KEY ((professional OR worker OR staff OR
practitioner OR technician OR technologist) OR doctor OR nurse OR physician OR intern OR internist OR
surgeon OR Pediatrician OR psychiatrist OR psychologist OR endocrinologist OR dentist OR odontologist)))
AND (PUBYEAR > 2001) OR (TITLE-ABS-KEY ('climate change' OR 'global warming' OR 'climate variability'
OR 'greenhouse effect' OR 'extreme weather' OR 'heat wave' OR drought OR flooding) AND TITLE-ABS-
KEY (health OR medical OR 'public health' OR healthcare OR clinic) AND TITLE-ABS-KEY (professional OR
worker OR staff OR practitioner OR technician OR technologist OR doctor OR nurse OR physician)) AND
(LIMIT-TO (PUBYEAR, 2018) OR LIMIT-TO (PUBYEAR, 2017) OR LIMIT-TO (PUBYEAR, 2016) OR LIMIT-TO
(PUBYEAR, 2015) OR LIMIT-TO (PUBYEAR, 2014) OR LIMIT-TO (PUBYEAR, 2013) OR LIMIT-TO (PUBYEAR,
2012) OR LIMIT-TO (PUBYEAR, 2011) OR LIMIT-TO (PUBYEAR, 2010) OR LIMIT-TO (PUBYEAR, 2009) OR
LIMIT-TO (PUBYEAR, 2008) OR LIMIT-TO (PUBYEAR, 2007) OR LIMIT-TO (PUBYEAR, 2006) OR LIMIT-TO
(PUBYEAR, 2005) OR LIMIT-TO (PUBYEAR, 2004) OR LIMIT-TO (PUBYEAR, 2003) OR LIMIT-TO (PUBYEAR,
2002))

Embase ('“Climate change':ti,ab,kw OR 'climate variability':ti,ab,kw OR 'global warming':ti,ab,kw OR 'greenhouse effect':ti,ab,kw OR 'ghge':ti,ab,kw OR 'extreme weather':ti,ab,kw OR 'heat wave':ti,ab,kw OR 'high temperature':ti, ab,kw OR 'drought':ti,ab,kw OR 'flooding'):ti,ab,kw) AND ((('health':ti,ab,kw OR 'medical':ti,ab,kw OR 'clinic':ti,ab,kw OR 'public health':ti,ab,kw OR 'healthcare':ti,ab,kw OR 'health care':ti,ab,kw) AND ('professional':ti,ab,kw OR 'worker':ti,ab,kw OR 'technician':ti,ab,kw OR 'technologist':ti,ab,kw OR 'staff':ti,ab,kw OR 'practitioner':ti,ab,kw OR 'officer':ti,ab,kw OR 'assistant':ti,ab,kw OR 'student':ti,ab,kw OR 'physician':ti,ab,kw OR 'physician assistant':ti,ab,kw OR 'general practitioner':ti,ab,kw OR 'general doctor':ti,ab,kw OR 'nurse':ti,ab,kw OR 'doctor':ti,ab,kw OR 'intern':ti,ab,kw OR 'internist':ti,ab,kw OR 'surgeon':ti,ab,kw OR 'Pediatrician' :ti,ab,kw OR 'psychiatrist':ti,ab,kw OR 'psychologist':ti,ab,kw OR 'endocrinologist':ti,ab,kw OR 'dentist':ti,ab,kw OR 'odontologist')):ti,ab,kw) AND [2002-]/py

$\mathrm{MeSH}$, Medical Subject Headings.

'challenge' will not be included in order to ensure the broadness of the captured literature. A preliminary search identified the earliest relevant study published in 2006. In the scoping review, we will wind 5 years ago to 2002. To capture as many relevant studies as possible, the search strategy will allow both MeSH terms and free text.

\section{Search syntaxes}

The key search terms of this study will be adapted to the requirements of different databases. The detailed search syntaxes are adapted from the review conducted by Osama $e t a l^{15}$ and outlined in table 3.

\section{Stage 3: Study selection and eligibility criteria}

We will use the EndNote software to remove duplicates of references searched by our research team.

The review process includes two stages: the first stage will screen titles and abstracts in English and the second stage will screen full text in English and Chinese. Two independent reviewers will screen titles, keywords and 
Table 4 Inclusion and exclusion criteria for the selection of articles

\begin{tabular}{ll}
\hline Inclusion criteria & Exclusion criteria \\
\hline Peer-reviewed articles & Book chapters and \\
without restriction on type of & grey literature (such as \\
publications, including original & conference proceedings, \\
quantitative and qualitative & dissertations and reports). \\
studies, reviews, viewpoints, & \\
editorials and commentaries &
\end{tabular}

Indexed in MEDLINE/PubMed,

Web of Science, Scopus and

Embase databases

Articles published since 2002

Focus on health professionals'

roles in climate change

\section{Publications in English and}

\section{Chinese}

No restriction to geographical

origin or population

abstracts of papers as recommended by Levac et $a l^{13}$ based on the inclusion and exclusion criteria determined by the research team. Table 4 showed inclusion and exclusion criteria for the selection of articles, which was adapted from Osama et al. ${ }^{15}$ Those that meet all of the eligibility criteria of inclusion will be subjected to full-text reading. Due to resource restrictions, we will not review the full text of articles published in a language other than English or Chinese unless their volume accounts for more than $20 \%$ of the identified abstracts. The two primary reviewers will resolve inconsistency in study eligibility, if exists, through discussions, before a third reviewer will be called on to assist in the paper selection process. The selection process including exclusion reasons will be recorded using a Preferred Reporting Items for Systematic Reviews and Meta-Analyses flow diagram to ensure replicability and transparency.

Stage 3 will be an iterative process, incorporating repeated attempts in search of the literature, adjustment of search strategies and selection of papers. ${ }^{13}$

\section{Stage 4: Charting the data}

A data charting form will be developed to guide data extraction from the eligible papers, including author, publication date, study type, study characteristics (location, climate change type, institutional setting [eg hospital, community health centre, CDC, medical college/university]), health professional characteristics, their knowledge, perception, behaviour and action details on climate change (age, gender, profession, interventions and measures, views and visions, willingness to act, resources support), and hurdles and challenges identified. The charting form will be reviewed and discussed by the research team prior to the implementation to ensure comprehensiveness and completeness.
The review will map the climate change and health professionals' knowledge, perceptions and practices. Two reviewers will conduct data extraction independently. The two forms will then be compared and reconciled through discussions. If necessary, a third reviewer will be involved before a single form containing the required data is finalised.

Further, a qualitative thematic analysis approach will be applied to categorise and present the key themes in our data. This is a common approach for scoping reviews. It involves coding the contents in the literature, identifying common themes across the literature and synthesising the logic link across the identified themes.

\section{Stage 5: Collating, summarising and reporting results}

Systematic scoping review studies provide an overview on a broad range of aspects of studies. ${ }^{12}$ Usually, the results will include a numerical synopsis of the amount and type of eligible studies and a narrative synthesis of the contents of included studies. We will develop a matrix framework for reviewing different aspects of the studies. For example, the roles of health professionals can be identified from microlevel, mesolevel and macrolevel. ${ }^{10} 16$ Under each level, there are different perceptions and actions.

The authors will discuss practice and policy implications of the findings, as well as the need for further studies in the future. ${ }^{13}$ To ensure a robust and holistic analysis, all different aspects of health professionals' preparedness regarding climate change will be collected. These will include, but not limited to knowledge, attitudes, practices and behaviours, interventions and programmes, development resources, and legal, organisational and financial support. It is believed that this method will allow us to determine the current situation of health professionals' preparedness in response to climate change, and to find the evidence gap(s) to inform the development of measures to enhance their knowledge and practice skills.

We will perform quality appraisal on the included studies using a rating framework developed by the research team based on the EQUATOR resources (www. equator-network.org/). We will make the quality rating framework and results available in the open access database. But we will not incorporate the quality appraisal in the review report because that is not customary for a scoping review.

\section{Stage 6: Consultation}

Public health professionals, professors and experts from the School of Public Health of Peking University, the Chinese CDC, the Department of Primary Care and Public Health of Imperial College (UK), London Hygiene and Tropical Medicine School (UK) and George Mason University (USA) will be consulted. They will offer valuable insights that are not captured through the literature review. At least one workshop will 
be organised supplemented by one-on-one meetings (face-to-face and online). Our research team has close collaboration with the above institutions, and these institutions have rich experiences and extensive studies in the field of capacity building, health professionals' knowledge, perceptions and actions on climate change. We will record and incorporate the results of consultations into the review report.

\section{Knowledge dissemination and translation}

Following completion of the scoping review, health professionals' perceptions, preparedness and actions regarding climate change over the last 16 years will be determined, yielding gaps as well as important policy, practice and research recommendations. Our recommendations will be tailored to the needs of health professionals, considering approaches for engaging and empowering health professionals. The scoping findings will be communicated and disseminated through reports and publications of the review.

Health professionals can play a vital role in communication and advocacy on climate change mitigation and adaptation. It is important that health professionals comprehensively perceive and actively communicate the health impacts of climate change. If knowledge of detrimental health effects is limited to concerned scientific communities only, health protection will not be adequate. Hence, through publication and circulation of our results on academic and lay websites, the authors aspire to develop an effective dissemination strategy to publicise recommendations to enhance health professionals' engagement.

We aim to utilise the knowledge achieved from this scoping review to develop an engagement and empowerment package for health professionals which will improve their knowledge and awareness, elicit individual behavioural change and enhance their capacity. ${ }^{1}$ We also intend for the collected evidence to support collective, high-level actions locally, nationally and internationally in order to address this great global health challenge of a changing climate. ${ }^{1}$

\section{CONCLUSION}

It is important to raise the awareness of and empower the health professionals in tackling climate change and its health risks. Around the world, we do not know about health professionals' engagement in mitigation activities or their preparedness to address the challenges of climate change. The findings of the review will enable the development of effective interventional measures and tools on the health impacts of climate change that will enhance the health professionals' engagement to address this global challenge.
Contributors $\mathrm{LY}$ wrote the first draft of the paper and initiated the project and data collection. $\mathrm{CL}$ and $\mathrm{CH}$ advised on study design, study methods and revised the draft paper. JH and DP advised on study methods and revised the draft paper. All authors approved the final version of the paper.

Funding This work was supported by the National Key R\&D Program of China [2018YFA0606200], the Guangdong Medical Science and Technology Foundation [A2016245], National Natural Science Foundation of China [71603292], Guangdong Natural Science Foundation [2016A030310162] and Guangdong Special Fund for Science and Technology Development [2017A070707002].

Competing interests None declared.

Patient consent for publication Not required.

Ethics approval No ethical approval is required for this literature-based study.

Provenance and peer review Not commissioned; externally peer reviewed.

Open access This is an open access article distributed in accordance with the Creative Commons Attribution Non Commercial (CC BY-NC 4.0) license, which permits others to distribute, remix, adapt, build upon this work non-commercially, and license their derivative works on different terms, provided the original work is properly cited, appropriate credit is given, any changes made indicated, and the use is non-commercial. See: http://creativecommons.org/licenses/by-nc/4.0/.

\section{REFERENCES}

1. Watts N, Adger WN, Agnolucci P, et al. Health and climate change: policy responses to protect public health. Lancet 2015;386:1861-914.

2. Watts N, Adger WN, Ayeb-Karlsson S, et al. The Lancet Countdown: tracking progress on health and climate change. Lancet 2017;389:1151-64.

3. Field CB, Barros VR, Dokken DJ, et al. Climate change 2014: impacts, adaptation, and vulnerability. Volume I: Global and Sectoral Aspects. Contribution of Working Group // to the Fifth Assessment Report of the Intergovernmental Panel on Climate Change. Cambridge and New York, 2014.

4. Frumkin $\mathrm{H}$, Hess J, Luber $\mathrm{G}$, et al. Climate change: the public health response. Am J Public Health 2008;98:435-45.

5. Huang C, Barnett AG, Xu Z, et al. Managing the health effects of temperature in response to climate change: challenges ahead. Environ Health Perspect 2013;121:415-9.

6. Benmarhnia T, Sottile MF, Plante C, et al. Variability in temperaturerelated mortality projections under climate change. Environ Health Perspect 2014;122:1293-8.

7. Ramanathan V, Haines A. Healthcare professionals must lead on climate change. BMJ 2016;355:i5245.

8. Roberts I, Stott R. Climate and Health Council executive. Doctors and climate change. BMJ 2010;341:c6357.

9. Maibach EW, Kreslake JM, Roser-Renouf C, et al. Do Americans understand that global warming is harmful to human health? Evidence from a national survey. Ann Glob Health 2015;81:396-409.

10. Costello A, Montgomery $\mathrm{H}$, Watts $\mathrm{N}$. Climate change: the challenge for healthcare professionals. BMJ 2013;347:f6060.

11. Sheehan MC, Fox MA, Kaye $C$, et al. Integrating health into local climate response: Lessons from the U.S. CDC Climate-Ready States And cities initiative. Environ Health Perspect 2017;125:094501.

12. Arksey H, O'Malley L. Scoping studies: towards a methodological framework. Int J Soc Res Methodol 2005;8:19-32.

13. Levac D, Colquhoun H, O'Brien KK. Scoping studies: advancing the methodology. Implement Sci 2010;5:69.

14. Daudt HM, van Mossel C, Scott SJ. Enhancing the scoping study methodology: a large, inter-professional team's experience with Arksey and O'Malley's framework. BMC Med Res Methodol 2013;13:48.

15. Osama T, Brindley D, Majeed A, et al. Teaching the relationship between health and climate change: a systematic scoping review protocol. BMJ Open 2018;8:e020330.

16. Shin GY, Manuel RJ. Healthcare professionals must "think globally, act locally" on climate change. BMJ 2016;355:i5686. 